\title{
Research Article \\ Complex Dynamics of Discrete SEIS Models with Simple Demography
}

\author{
Hui Cao, ${ }^{1}$ Yicang Zhou, ${ }^{1}$ and Baojun Song ${ }^{2}$ \\ ${ }^{1}$ Department of Applied Mathematics, Xi'an Jiaotong University, Xi'an 710049, China \\ ${ }^{2}$ Department of Mathematical Sciences, Montclair State University, Montclair, NJ 07043, USA \\ Correspondence should be addressed to Hui Cao, caohui0103@163.com
}

Received 29 May 2011; Revised 23 August 2011; Accepted 23 August 2011

Academic Editor: Elmetwally Elabbasy

Copyright (c) 2011 Hui Cao et al. This is an open access article distributed under the Creative Commons Attribution License, which permits unrestricted use, distribution, and reproduction in any medium, provided the original work is properly cited.

\begin{abstract}
We investigate bifurcations and dynamical behaviors of discrete SEIS models with exogenous reinfections and a variety of treatment strategies. Bifurcations identified from the models include period doubling, backward, forward-backward, and multiple backward bifurcations. Multiple attractors, such as bistability and tristability, are observed. We also estimate the ultimate boundary of the infected regardless of initial status. Our rigorously mathematical analysis together with numerical simulations show that epidemiological factors alone can generate complex dynamics, though demographic factors only support simple equilibrium dynamics. Our model analysis supports and urges to treat a fixed percentage of exposed individuals.
\end{abstract}

\section{Introduction}

Pure demographic (or ecological) discrete models can have very complicated dynamics. It is well known that the logistic model $x_{n+1}=r x_{n}\left(1-x_{n} / K\right)$, Ricker's model $x_{n+1}=x_{n} e^{r-x_{n} / K}$ [1-3], and Hassell model $x_{n+1}=\lambda x_{n}\left(1+a x_{n}\right)^{-b}$ [4] all have complex dynamics through the mechanics of period-doubling bifurcation. When adding epidemiological effects to these demographic models to describe the dynamics of an infectious disease, it is not surprising at all that the complex dynamics still retain $[5,6]$. Yet an interesting question is that if adding epidemiological effects to a simple demographic model that has only trivial dynamics, for example, $x_{n+1}=\Lambda+\mu x_{n}$, is it possible to observe complex dynamics? Our work in this paper finds a quite positive answer by bifurcation analysis. It is shown that the chaotic dynamics is possible.

The bifurcation approach has been extensively utilized in theoretical epidemiology. The use of equilibrium bifurcation to investigate the dynamical behavior of continuous epidemic models has been successful, for instance, the work in [7-15]. Meanwhile, discrete epidemic models have gained more popularity $[5,6,16-25]$, since epidemiological data are 
usually collected at discrete times and it becomes easier to compare data with models. We will use the bifurcation approach to analyze discrete SEIS epidemic models in this paper.

The appearance of multiple attractors enhances uncertainty of outcomes because of the lack of information to the initial epidemiological status, even though the domain of attractions of each attractor is fully depicted. In this case, it would be necessary to estimate the prevalence regardless of the initial data.

The management measures to completely wipe out an infectious disease may be too costly, far beyond the capability of any society. A practical and feasible way is to have an infectious disease under control, that is, to keep the number of the infectious individuals under a certain level. Thus, estimating an upper bound of the prevalence appears necessary. A large pool of excellent researches have focused on the lower bound of prevalence in the application of permanence theory [26-29], while there are relatively few studies on the upper boundary of infections when a disease persists, especially when multiple attractors exist. In this paper, we also estimate ultimate boundaries, which are given in terms of the basic reproductive number if the disease persists. We believe, in some sense, that an ultimate boundary estimation for an infectious disease is more significant than a basic reproductive number, especially when multiple attractors appear.

Our mathematical models are presented in Section 2. In Section 3, we compute the reproductive number and simplify our models. In Section 4, we analyze the different bifurcations of the models under different treatment strategies and with or without considering exogenous reinfections. Numerical simulations are carried out to test and confirm each type of bifurcations. The ultimate boundaries of the latent and the infectious are obtained under some sufficient conditions. Section 5 includes some concluding remarks and discussions.

\section{The Model}

Our models could be built within the context of the dynamical and epidemiological study of tuberculosis, where both the exogenous reinfections and the different treatment strategies play an important role. Existing studies have shown that the exogenous re-infection of the latent individuals increases the risk of developing into the active disease [20, 30, 31]. The effect of the treatment for the latent individuals on the dynamics of disease has been investigated using continuous models $[32,33]$. Here, we will investigate the treatment effect of the latent individuals on the dynamics of the disease using discrete epidemic models. The models will incorporate two distinct features: exogenous re-infection and flexible treatment strategies.

In modeling the treatment for the latent and the infectious individuals, we should consider both the prevalence of the disease and availability of a budget. The treatment rate for infectious ones is rather easy; we simply take a linear function, as has been typically used. However, a practice of treating the latent individuals, like inactive cases of tuberculosis, is highly theoretic, mainly because it is hard to find asymptomatic individuals without massscreening. When the pool of the latent individuals is small, it is reasonable to assume that the treatment rate is proportional to the number of the latent individuals (a linear function of the number of the latent individuals). However, if the pool is big, such as the case of tuberculosis in India, China, Eastern Europe, or South Sahara, taking a linear function as a treatment rate would be inappropriate due to the limited budgets. We cannot provide treatment to all individuals with latent tuberculosis, since the people living with latent tuberculosis are approximately one-third of the total population. The other obvious reason is our inability 
of identifying asymptomatic individuals. We have to design the treatment strategy within our reach of case finding and budgets. It then becomes more plausible to assume a constant treatment rate when the pool of latent individuals is large, as has been used in $[13,15]$, for instance. Overall, treatment strategies should be designed by considering both the prevalence of the disease and available budget. Part of our goal in this paper is to compare these strategies.

The total population is epidemiologically divided into classes of susceptible, latent, and infectious. Let $S(t)$ the number of susceptible individuals at time $t, E(t)$ be the number of latent individuals, and $I(t)$ the number of infectious individuals. $N(t)=S(t)+E(t)+I(t)$ is the total population size. We assume that recovered individuals by successful treatment do not acquire immunity and will become member of susceptible compartment. Hence our model is of SEIS type.

Susceptible individuals may get infected and enter the latent compartment. The survived latent individuals will experience one of the three mutually exclusive events to leave the latent compartment, getting exogenous re-infection through contacting with infectious individuals, or naturally progressing into infectious class, or receiving the treatment. These three events happen randomly. The respective probabilities for exogenous re-infection, natural progression, and receiving the treatment are $k_{1}, k_{2}$, and $k_{3}$ with $k_{1}+k_{2}+k_{3}=1$. Incorporating the exogenous reinfections and treatments into the models in [20, 21], our model framework takes the following form:

$$
\begin{aligned}
S(t+1)= & \Lambda+p S(t) G_{1}\left(\frac{I(t)}{N(t)}\right)+p H(E(t))+p \gamma I(t), \\
E(t+1)= & p S(t)\left(1-G_{1}\left(\frac{I(t)}{N(t)}\right)\right)+p k_{1} E(t) G_{2}\left(\frac{I(t)}{N(t)}\right) \\
& +p k_{2}(1-\alpha) E(t)+p\left(k_{3} E(t)-H(E(t))\right), \\
I(t+1)= & p k_{1} E(t)\left(1-G_{2}\left(\frac{I(t)}{N(t)}\right)\right)+p \alpha k_{2} E(t)+p(1-\gamma) I(t),
\end{aligned}
$$

where $\Lambda$ is the recruitment rate into the population, $p$ is the survival probability, and $\gamma$ is the probability that an infectious individual recovers successfully. $\alpha$ is the conditional probability that a latent individual becomes infectious successfully given that the natural progression happens. $1-G_{1}$ is the probability that susceptible individuals become infected. $1-G_{2}$ is the conditional probability that a latent individual gets re-infected successfully given that the exogenous re-infection happens. The probabilities of not becoming infected are $G_{1}$ and $G_{2}$ that are considered as functions of the prevalence $I(t) / N(t) \cdot \exp \left(-\beta_{j} I(t) / N(t)\right)$ and $1-\left(\beta_{j} I(t) / N(t)\right)$ have been used for the probabilities of not becoming infected $[20,21]$. We choose $G_{j}(I(t) / N(t))=1-\left(\beta_{j} I(t) / N(t)\right), j=1,2$, where $\beta_{j}$ characterizes the disease transmission probability.

In model (2.1), $H(E(t))$ is the successful treatment rate of the latent individuals and $k_{3} E(t)-H(E(t))$ is the failure rate of the treatment. We consider two different types of treatment strategies for the latent individuals:

$$
H(E(t))=m k_{3} E(t) \quad \text { or } \quad H(E(t))=m k_{3} \begin{cases}E(t), & E(t) \leq E^{0}, \\ E^{0}, & E(t)>E^{0}\end{cases}
$$


where $m$ is the conditional probability that a latent individual is treated successfully given that the individual receives the treatment and $E^{0}$ is the cutting point of treatment determined by policy makers. Both $m$ and $E^{0}$ should rely on the capability of finding latent individuals and budgetary issues.

We summarize some important terms in model (2.1) here. From time $t$ to time $t+1$, the number of the susceptibles who get infected and become latent individuals is $p S(t)(1-$ $\left.G_{1}(I(t) / N(t))\right)$; the number of the latent who get re-infected and become infectious is $p k_{1} E(t)\left(1-G_{2}(I(t) / N(t))\right)$; the number of the latent who naturally progress into the infectious compartment is $p k_{2} \alpha E(t)$; the number of the latent who get recovered by successful treatment and become susceptible is $p H(E(t))$; the number of the infectious who get recovered and become susceptible is $\operatorname{pr} I(t)$. The number of the latent individuals at time $t$ that remain as latent ones at time $t+1$ is $p k_{1} E(t) G_{2}(I(t) / N(t))+p k_{2}(1-\alpha) E(t)+p\left(k_{3} E(t)-H(E(t))\right)$. Similarly, the number of infectious individuals who do not change their epidemiological status from $t$ to $t+1$ is counted by $p(1-\gamma) I(t)$.

\section{Basic Reproductive Number and Reduced Model}

Firstly we explicitly write our models for two specific treatment regimens. In the case of proportional treatment rate of the latent individuals, the treatment function is linear, $H(E(t))=m k_{3} E(t)$, and model (2.1) can be rewritten as follows:

$$
\begin{gathered}
S(t+1)=\Lambda+p S(t)\left(1-\frac{\beta_{1} I(t)}{N(t)}\right)+p m k_{3} E(t)+p \gamma I(t), \\
E(t+1)=p S(t) \frac{\beta_{1} I(t)}{N(t)}+p k_{1} E(t)\left(1-\frac{\beta_{2} I(t)}{N(t)}\right)+p(1-\alpha) k_{2} E(t)+p(1-m) k_{3} E(t), \\
I(t+1)=p k_{1} E(t) \frac{\beta_{2} I(t)}{N(t)}+p \alpha k_{2} E(t)+p(1-\gamma) I(t) .
\end{gathered}
$$

On the other hand, in the case of limited resources, the treatment function of latent individuals is a piecewise function,

$$
H(E(t))= \begin{cases}m k_{3} E(t), & E(t) \leq E^{0} \\ m_{0}, & E(t)>E^{0}\end{cases}
$$

where $m_{0}=m k_{3} E^{0}$. Model (2.1) takes the following form:

$$
\begin{gathered}
S(t+1)=\Lambda+p S(t)\left(1-\frac{\beta_{1} I(t)}{N(t)}\right)+p k_{3} E(t)-T(E(t))+p \gamma I(t) \\
E(t+1)=p S(t) \frac{\beta_{1} I(t)}{N(t)}+p k_{1} E(t)\left(1-\frac{\beta_{2} I(t)}{N(t)}\right)+p(1-\alpha) k_{2} E(t)+T(E(t)), \\
I(t+1)=p k_{1} E(t) \frac{\beta_{2} I(t)}{N(t)}+p \alpha k_{2} E(t)+p(1-\gamma) I(t)
\end{gathered}
$$


where

$$
T(E(t))= \begin{cases}p(1-m) k_{3} E(t), & \text { if } E(t) \leq E^{0}, \\ p\left(k_{3} E(t)-m_{0}\right), & \text { if } E(t)>E^{0} .\end{cases}
$$

The disease-free equilibrium, $P_{0}(\Lambda /(1-p), 0,0)$, and the basic reproductive number of models (3.1) and (3.3) are identical. Applying the approach in [34] to our model, we have

$$
\mathcal{F}=\left(\begin{array}{c}
p S(t) \frac{\beta_{1} I(t)}{N(t)} \\
p k_{1} E(t) \frac{\beta_{2} I(t)}{N(t)} \\
0
\end{array}\right), \quad \mho=\left(\begin{array}{c}
p\left(1-k_{1} \frac{\beta_{2} I(t)}{N(t)}-\alpha k_{2}-m k_{3}\right) E(t) \\
p \alpha k_{2} E(t)+p(1-\gamma) I(t) \\
\Lambda+p S(t)\left(1-\frac{\beta_{1} I(t)}{N(t)}\right)+p m k_{3} E(t)+p \gamma I(t)
\end{array}\right)
$$

At the disease-free equilibrium, the straight forward calculation yields $F=\left(\begin{array}{cc}0 & p \beta_{1} \\ 0 & 0\end{array}\right)$ and $V=$ $\left(\begin{array}{cc}p-p \alpha k_{2}-p m k_{3} & 0 \\ p \alpha k_{2} & p(1-\gamma)\end{array}\right)$. Therefore, the basic reproductive number of model (2.1) is

$$
R_{0}=\frac{p^{2} \beta_{1} \alpha k_{2}}{(1-p(1-\gamma))\left(1-p+p \alpha k_{2}+p m k_{3}\right)} .
$$

Rearrange terms in the expression of $R_{0}$ as

$$
R_{0}=\left(\frac{p \beta_{1}}{1-p(1-\gamma)}\right)\left(\frac{p \alpha k_{2}}{1-p+p \alpha k_{2}+p m k_{3}}\right) \text {. }
$$

Each term in $R_{0}$ has clear epidemiological interpretation. $1 /(1-p(1-\gamma))$ is the average infection period. $p \beta_{1} /(1-p(1-\gamma))$ is average new cases generated by a typical infectious member in the entire infection period. $p \alpha k_{2} /\left(1-p+p \alpha k_{2}+p m k_{3}\right)$ is the proportion that latent individuals become infectious by "natural" progression.

It is known that the disease-free equilibrium $P_{0}$ of (2.1) is locally asymptotically stable if $R_{0}<1$ and unstable if $R_{0}>1$ (see [34, Theorem 2.1]). We summarize stability results in terms of $R_{0}<1$ as in the following theorem.

Theorem 3.1. The disease-free equilibrium $P_{0}$ of model (2.1) is locally asymptotically stable if $R_{0}<1$ and unstable if $R_{0}>1$.

Since we do not consider the disease-induced death rate, the total population size is governed by an extremely simple equation. In fact, by adding all equations in model (2.1), we obtain the following equation for the total population $N(t)=S(t)+E(t)+I(t)$ :

$$
N(t+1)=\Lambda+p N(t)
$$


One can see that $N^{*}=\Lambda /(1-p)$ is a global attractor for (3.8). Using limiting equations and the $\lim _{t \rightarrow \infty} N(t)=N^{*}$ [35], we, respectively, reduce the three-dimensional systems (3.1) and (3.3) into two-dimensional ones

$$
\begin{aligned}
E(t+1)= & p\left(N^{*}-E(t)-I(t)\right) \frac{\beta_{1} I(t)}{N^{*}}+p k_{1} E(t)\left(1-\frac{\beta_{2} I(t)}{N^{*}}\right) \\
& +p(1-\alpha) k_{2} E(t)+p(1-m) k_{3} E(t), \\
I(t+1)= & p k_{1} E(t) \frac{\beta_{2} I(t)}{N^{*}}+p \alpha k_{2} E(t)+p(1-\gamma) I(t), \\
E(t+1)= & p\left(N^{*}-E(t)-I(t)\right) \frac{\beta_{1} I(t)}{N^{*}}+p k_{1} E(t)\left(1-\frac{\beta_{2} I(t)}{N^{*}}\right) \\
& +p(1-\alpha) k_{2} E(t)+T(E(t)), \\
I(t+1)= & p k_{1} E(t) \frac{\beta_{2} I(t)}{N^{*}}+p \alpha k_{2} E(t)+p(1-\gamma) I(t) .
\end{aligned}
$$

In the rest of the paper, we analyze the dynamic behavior of the limiting system (3.9) and (3.10) under different treatment strategies.

\section{Analysis}

In this section, we study the bifurcation and stability of equilibrium of model (3.9) and (3.10). Numerical simulations are also presented to demonstrate the theoretical results.

\subsection{Without Exogenous Reinfections}

The SEIS model is the simplest one if there is no exogenous re-infection and the treatment rate takes a linear form. For this case, $k_{1}=0, H(E)=m k_{3} E$, and model (3.9) is reduced to

$$
\begin{gathered}
E(t+1)=p\left(N^{*}-E(t)-I(t)\right) \frac{\beta_{1} I(t)}{N^{*}}+p(1-\alpha) k_{2} E(t)+p(1-m) k_{3} E(t) \\
I(t+1)=p \alpha k_{2} E(t)+p(1-\gamma) I(t) .
\end{gathered}
$$

The disease-free equilibrium of $(4.1)$ is $P_{1}^{0}\left(E_{1}^{0}, I_{1}^{0}\right)=(0,0)$ and the endemic equilibrium $P_{1}^{*}\left(E_{1}^{*}, I_{1}^{*}\right)$ exists if $R_{0}>1$, where $E_{1}^{*}=\left((1-p(1-\gamma)) N^{*} /\left(1-p(1-\gamma)+p k_{2} \alpha\right)\right)\left(1-\left(1 / R_{0}\right)\right)$ and $I_{1}^{*}=p k_{2} \alpha E_{1}^{*} /(1-p(1-\gamma))$.

\subsubsection{Global Stability of $P_{1}^{0}$}

The global stability of the disease-free equilibrium $P_{1}^{0}\left(E_{1}^{0}, I_{1}^{0}\right)=(0,0)$ of $(4.1)$ is given in Theorem 4.1.

Theorem 4.1. If $R_{0}<1$, then the disease-free equilibrium $P_{1}^{0}$ of (4.1) is globally asymptotically stable. 
Proof. Define $F:\left[0, N^{*}\right] \times\left[0, N^{*}\right] \rightarrow\left[0, N^{*}\right] \times\left[0, N^{*}\right]$ by

$$
F(E, I)=p\left(\left(N^{*}-E-I\right) \frac{\beta_{1} I}{N^{*}}+(1-\alpha) k_{2} E+(1-m) k_{3} E, \alpha k_{2} E+(1-\gamma) I\right)
$$

Obviously, $F$ is the mapping derived by system $(4.1)$, and $(0,0)$ is a fixed point of $F$. Linear function $V(E, I)=E+\left(p \beta_{1} /(1-p(1-\gamma))\right) I$ on $\left[0, N^{*}\right] \times\left[0, N^{*}\right]$ is continuous and positive definite with respect to $(0,0)$. Therefore, $V$ is a Lyapunov function on the domain of $F$. For any $(E, I) \in\left[0, N^{*}\right] \times\left[0, N^{*}\right]$,

$$
\begin{aligned}
\Delta V(E, I)= & p\left(N^{*}-E-I\right) \frac{\beta_{1} I}{N^{*}}+p(1-\alpha) k_{2} E+p(1-m) k_{3} E \\
& +\frac{p \beta_{1}}{1-p(1-\gamma)}\left(p \alpha k_{2} E+p(1-\gamma) I\right)-E-\frac{p \beta_{1}}{1-p(1-\gamma)} I \\
\leq & p \beta_{1} I-\left(1-p(1-\alpha) k_{2}-p(1-m) k_{3}\right) E+\frac{p^{2} \beta_{1} \alpha k_{2} E}{1-p(1-\gamma)}+p \beta_{1} I \\
= & \left(1-p(1-\alpha) k_{2}-p(1-m) k_{3}\right)\left(R_{0}-1\right) E \leq 0 .
\end{aligned}
$$

Hence, if $R_{0}<1$, then $\Delta V(E, I)<0$ holds for $(E, I) \in\left(0, N^{*}\right] \times\left(0, N^{*}\right]$. It follows from Theorem 4.22 in [36] that $P_{1}^{0}$ is globally asymptotically stable.

Theorem 4.1 ensures that the disease-free equilibrium $P_{0}(\Lambda /(1-p), 0,0)$ of model (3.1) is globally stable as $R_{0}<1$ and $k_{1}=0$.

\subsubsection{Stability of $P_{1}^{*}$}

The stability of the endemic equilibrium is given in Theorem 4.2 .

Theorem 4.2. If $1<R_{0}<1+\sigma$, then the endemic equilibrium $P_{1}^{*}$ of (4.1) is asymptotically stable, where

$$
\sigma=\frac{2 p\left(1-\alpha k_{2}-m k_{3}+(1-\gamma)\right)\left(1-p(1-\gamma)+p \alpha k_{2}\right)}{\left(1+p(1-\gamma)-p \alpha k_{2}\right)(1-p(1-\gamma))\left(1-p+p \alpha k_{2}+p m k_{3}\right)}>0
$$

Proof. The linearization matrix of (4.1) at the endemic equilibrium $P_{1}^{*}$ is

$$
J_{1}=\left(\begin{array}{cc}
-\frac{p \beta_{1}}{N^{*}} I_{1}^{*}+p(1-\alpha) k_{2}+p(1-m) k_{3} & \frac{p \beta_{1}}{N^{*}}\left(N^{*}-E_{1}^{*}-2 I_{1}^{*}\right) \\
p \alpha k_{2} & p(1-\gamma)
\end{array}\right)
$$

The corresponding characteristic equation is

$$
f_{1}(\lambda)=\lambda^{2}-b_{1} \lambda+b_{2}=0
$$


where

$$
\begin{aligned}
b_{1}= & p-p \alpha k_{2}-p m k_{3}+p(1-\gamma)-\frac{(1-p(1-\gamma))\left(1-p+p \alpha k_{2}+p m k_{3}\right)\left(R_{0}-1\right)}{1-p(1-\gamma)+p \alpha k_{2}}, \\
b_{2}= & p(1-\gamma)\left(p-p \alpha k_{2}-p m k_{3}\right)-(1-p(1-\gamma))\left(1-p+p \alpha k_{2}+p m k_{3}\right) \\
& +\frac{\left(p \alpha k_{2}-p(1-\gamma)\right)(1-p(1-\gamma))\left(1-p+p \alpha k_{2}+p m k_{3}\right)\left(R_{0}-1\right)}{1-p(1-\gamma)+p \alpha k_{2}}
\end{aligned}
$$

We claim $\left|b_{2}\right|<1$. When $p \alpha k_{2}=p(1-\gamma)$, we have $b_{2}=p(1-\gamma)\left(p-p \alpha k_{2}-p m k_{3}\right)-(1-p(1-$ $\gamma))\left(1-p+p \alpha k_{2}+p m k_{3}\right)$, and $\left|b_{2}\right|<1$ holds. When $p \alpha k_{2} \neq p(1-\gamma)$, because of

$$
\begin{aligned}
& \frac{2 p\left(1-\alpha k_{2}-m k_{3}+(1-\gamma)\right)}{\left(1+p(1-\gamma)-p \alpha k_{2}\right)} \\
& \quad<\min \left\{\frac{2-p+p \alpha k_{2}+p m k_{3}-p(1-\gamma)}{p \alpha k_{2}-p(1-\gamma)}, \frac{p\left(1-\alpha k_{2}-m k_{3}+(1-\gamma)\right)}{p(1-\gamma)-p \alpha k_{2}}\right\},
\end{aligned}
$$

we obtain that $\left|b_{2}\right|<1$ holds if $1<R_{0}<1+\sigma$. Furthermore, we have

$$
\begin{aligned}
& f_{1}(1)=1-b_{1}+b_{2}=(1-p(1-\gamma))\left(1-p+p \alpha k_{2}+p m k_{3}\right)\left(R_{0}-1\right) \\
& (-1)^{2} f_{1}(-1)=1+b_{1}+b_{2}=2\left(p-p \alpha k_{2}-p m k_{3}+p(1-\gamma)\right) \\
& \quad-\frac{\left(1+p(1-\gamma)-p \alpha k_{2}\right)(1-p(1-\gamma))\left(1-p+p \alpha k_{2}+p m k_{3}\right)\left(R_{0}-1\right)}{1-p(1-\gamma)+p \alpha k_{2}} .
\end{aligned}
$$

It is clear that $f_{1}(1)>0$ holds if $R_{0}>1$ and

$$
(-1)^{2} f_{1}(-1)=\frac{\sigma\left(1+\sigma-R_{0}\right)}{\left(1+p(1-\gamma)-p \alpha k_{2}\right)(1-p(1-\gamma))\left(1-p+p \alpha k_{2}+p m k_{3}\right)}>0
$$

holds if $R_{0}<1+\sigma$. Therefore, if $1<R_{0}<1+\sigma$, we have $f_{1}(1)>0,(-1)^{2} f_{1}(-1)>0$, and $\left|b_{2}\right|<1$. It follows from the Jury criteria that the endemic equilibrium $P_{1}^{*}\left(E_{1}^{*}, I_{1}^{*}\right)$ of $(4.1)$ is asymptotically stable.

It follows from Theorem 4.2 that when exogenous re-infection is not included $\left(k_{1} \beta_{2}=\right.$ $0)$ the endemic equilibrium $\left.P_{1}^{*}(\Lambda /(1-p))-E_{1}^{*}-I_{1}^{*}, E_{1}^{*}, I_{1}^{*}\right)$ of model (3.1) is asymptotically stable if $1<R_{0}<1+\sigma$. Since the Jury criteria is the necessary and sufficient condition for the local stability of the equilibrium, the endemic equilibrium $P_{1}^{*}$ of (3.1) becomes unstable if 


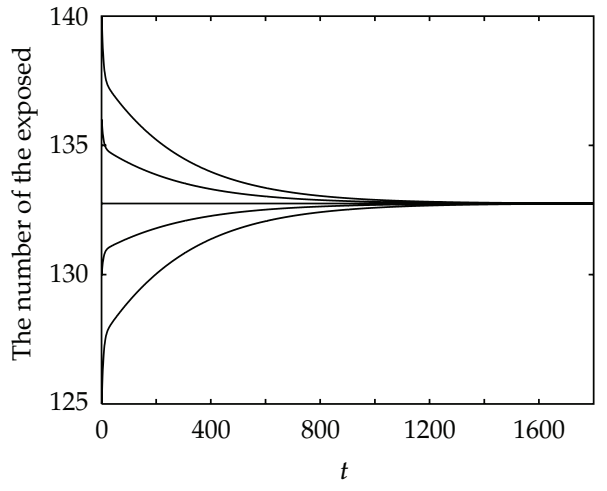

(a)

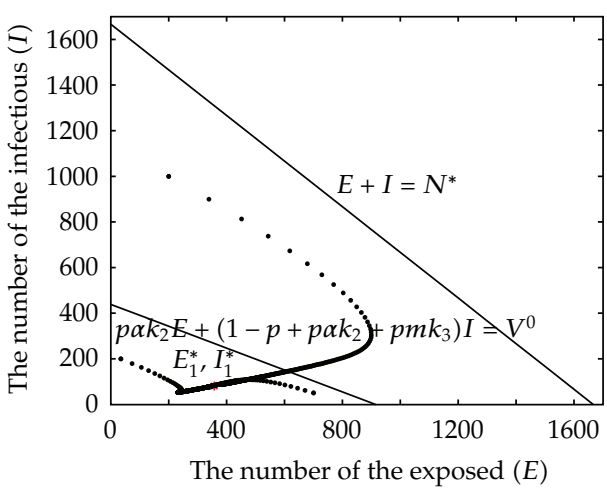

(b)

Figure 1: The stability and the attraction of the endemic equilibrium of (3.1): (a) the endemic equilibrium and its stability and (b) the attraction area.

$R_{0} \geq 1+\sigma$. We summarize Theorems 4.1 and 4.2 for model (3.1) without exogenous reinfection as follows.

(1) The disease-free equilibrium $P_{0}(\Lambda /(1-p), 0,0)$ is globally stable as $R_{0}<1$.

(2) When $R_{0}>1, P_{0}(\Lambda /(1-p), 0,0)$ becomes unstable and endemic equilibrium $P_{1}^{*}$ is born. The endemic $P_{1}^{*}$ is locally asymptotically stable if $1<R_{0}<1+\sigma$.

(3) When $R_{0} \geq 1+\sigma, P_{1}^{*}$ becomes unstable.

Theorems 4.1 and 4.2 state that a forward bifurcation takes place at $R_{0}=1$. This is consistent with continuous dynamical models for tuberculosis when exogenous re-infection is not considered [30,31].

Although the expression of $\sigma$ is complicated, the condition in Theorem 4.2 is easy to check since $\sigma$ is independent of $\beta_{1}$ while $R_{0}$ depends on $\beta_{1}$ linearly. For example, given a set of the parameter values, $p=0.994, \Lambda=10, \alpha=0.03, \gamma=0.1, m=0.1, k_{1}=0, k_{2}=0.8$, and $k_{3}=0.2$, one can determine if the endemic equilibrium is stable or not. Numerical simulations suggest that the endemic equilibrium of model (3.1) may be globally asymptotically stable when $1<$ $R_{0}<1+\sigma$, as shown in Figure 1 (a) for $\beta_{1}=0.245$. When $\beta_{1}=10.972015$, we have $R_{0}=49.63168$ and $1+\sigma=49.63168$. The endemic equilibrium of model (4.1) loses the stability when $\beta_{1}$ is greater than 10.972015, and there is a 2-period solution when $\beta_{1} \in[10.972015,11.109)$. For example, if we take $\beta_{1}=11$, then $R_{0}=49.75827$, and $1+\sigma=49.63168$. The 2-period solution is ((838.46721, 189.77679), (1591.60886,360.24119)). Although model (4.1) has a period solution of period 2 if the endemic equilibrium of model (4.1) is unstable, the periodic solution may not be realistic since model (4.1) is the limiting system of model (3.1) as $k_{1} \beta_{2}=0$, and the numerical computation shows that the corresponding periodic solution of model (3.1) is ((838.46721, 189.77679, 638.42267), (1591.60886, 360.24119,-285.18339)). Obviously, one of $S^{*}$ is negative, which hints that the period solution of model (3.1) can be ruled out if we confine all solutions as positive. In fact, $1-G_{1}(I(t) / N(t))=\beta_{1} I(t) / N(t)$ means that $\beta_{1}$ should not be bigger, and we should chose $\beta_{1}$ such that $0 \leq 1-G_{1}(I(t) / N(t)) \leq 1$. Otherwise, not only does $1-G_{1}(I(t) / N(t))$ lose its meaning but also the number of the susceptible individuals has negative values. 


\subsubsection{Ultimate Boundary}

We now estimate an ultimate boundary to the model (4.1) when $R_{0}>1$.

Theorem 4.3. If $0<\beta_{1} \leq 1$ and $1<R_{0}<1+(1 /(1-p(1-\gamma)))$, then the solutions of model (4.1) are bounded by

$$
\begin{gathered}
\limsup _{t \rightarrow \infty}\left(\alpha k_{2} E(t)+\left(1-p+p \alpha k_{2}+p m k_{3}\right) I(t)\right) \leq\left(1-p+p \alpha k_{2}+p m k_{3}\right) N^{*}\left(1-\frac{1}{R_{0}}\right), \\
\limsup _{t \rightarrow \infty} E(t) \leq\left(1+\frac{1-p+p m k_{3}}{p \alpha k_{2}}\right) N^{*}\left(1-\frac{1}{R_{0}}\right), \quad \limsup _{t \rightarrow \infty} I(t) \leq N^{*}\left(1-\frac{1}{R_{0}}\right) .
\end{gathered}
$$

Proof. Let us consider the value of $V(E(t), I(t))$ for any $(E(t), I(t)) \in \Omega$, where

$$
\Omega=\left\{(E(t), I(t)) \mid E(t) \geq 0, I(t) \geq 0, E(t)+I(t) \leq N^{*}\right\} .
$$

Define

$$
\begin{aligned}
& V(E, I)=p \alpha k_{2} E+\left(1-p+p \alpha k_{2}+p m k_{3}\right) I \\
& V_{0}=\left(1-p+p \alpha k_{2}+p m k_{3}\right) N^{*}\left(1-\frac{1}{R_{0}}\right)
\end{aligned}
$$

Then,

$$
\begin{aligned}
\Delta V & =V(E(t+1), I(t+1))-V(E(t), I(t)) \\
& =q\left(R_{0}-1-\frac{R_{0}}{N^{*}} \frac{1-p+p \alpha k_{2}+p m k_{3}}{1-p+p \alpha k_{2}+p m k_{3}}(E(t)+I(t))\right) I(t) \\
& \leq q\left(R_{0}-1-\frac{R_{0}}{N^{*}} \frac{V(E(t), I(t))}{1-p+p \alpha k_{2}+p m k_{3}}\right) I(t), \\
& =\frac{(1-p(1-\gamma)) R_{0}}{N^{*}}\left(V_{0}-V(E(t), I(t))\right) I(t),
\end{aligned}
$$

where $q=\left(1-p(1-\gamma)\left(1-p+p \alpha k_{2}+p m k_{3}\right)\right)$. Therefore, $\Delta V \leq 0$ holds if $V \geq V_{0}$.

If $V\left(E\left(t_{0}\right), I\left(t_{0}\right)\right)=p \alpha k_{2} E\left(t_{0}\right)+\left(1-p+p \alpha k_{2}+p m k_{3}\right) I\left(t_{0}\right) \leq V_{0}$ for a $t_{0} \geq 0$, then we claim that $V(E(t), I(t)) \leq V_{0}$ for all $t \geq t_{0}$. In fact, let $V\left(E\left(t_{0}\right), I\left(t_{0}\right)\right)=V_{0}-\varepsilon$ with some $\varepsilon \geq 0$. Then, the inequality $V\left(E\left(t_{0}\right), I\left(t_{0}\right)\right)=V_{0}-\varepsilon \leq V_{0}$ yields

$$
I\left(t_{0}\right) \leq \frac{V_{0}}{1-p+p \alpha k_{2}+p m k_{3}}=N^{*}\left(1-\frac{1}{R_{0}}\right) .
$$


The inequality in (4.14) and the condition in Theorem 4.3 as well as the inequality for $I\left(t_{0}\right)$ imply

$$
\begin{aligned}
\Delta V & =V\left(E\left(t_{0}+1\right), I\left(t_{0}+1\right)\right)-V\left(E\left(t_{0}\right), I\left(t_{0}\right)\right) \\
& \leq \frac{(1-p(1-\gamma)) R_{0}}{N^{*}}\left(V_{0}-V\left(E\left(t_{0}\right), I\left(t_{0}\right)\right)\right) I\left(t_{0}\right), \\
& \leq(1-p(1-\gamma)) R_{0}\left(V_{0}-V\left(E\left(t_{0}\right), I\left(t_{0}\right)\right)\right)\left(1-\frac{1}{R_{0}}\right) \\
& \leq(1-p(1-\gamma))\left(R_{0}-1\right) \varepsilon \leq \varepsilon .
\end{aligned}
$$

The inequality in (4.16) leads to

$$
V\left(E\left(t_{0}+1\right), I\left(t_{0}+1\right)\right) \leq V\left(E\left(t_{0}\right), I\left(t_{0}\right)\right)+\varepsilon=V_{0}-\varepsilon+\varepsilon=V_{0}
$$

Then, our claim is followed from the mathematical induction.

If $V\left(E\left(t_{0}\right), I\left(t_{0}\right)\right)=p \alpha k_{2} E\left(t_{0}\right)+\left(1-p+p \alpha k_{2}+p m k_{3}\right) I\left(t_{0}\right)>V_{0}$ for a $t_{0} \geq 0$, then there are two cases that we have to deal with.

Case 1. There exists an integer $t_{1}>t_{0}$ such that $V\left(E\left(t_{1}\right), I\left(t_{1}\right) \leq V_{0}\right.$.

Case 2. The inequality $V(E(t), I(t))>V_{0}$ holds for all $t \geq t_{0}$.

For Case 1 we can prove that $V(E(t), I(t)) \leq V_{0}$ for all $t \geq t_{1}$ by the similar argument adopted for the case $V\left(E\left(t_{0}\right), I\left(t_{0}\right)\right) \leq V_{0}$. The boundary of $V(E(t), I(t)) \leq V_{0}$ for $t>t_{1}$ gives rise to $\limsup _{t \rightarrow \infty} V(E(t), I(t)) \leq V_{0}$. For Case 2, the fact that $\Delta V(E(t), I(t)) \leq 0$ for $V(E(t), I(t))>V_{0}$ leads to that the $V(E(t), I(t))$ is a monotonic decreasing sequence. Hence, the limit $\lim _{t \rightarrow \infty} V(E(t), I(t))$ exits. We claim that $\lim _{t \rightarrow \infty} V(E(t), I(t)) \leq V_{0}$. Otherwise, there exists $V^{*}>V_{0}$, such that $\lim _{t \rightarrow \infty} V(E(t), I(t))=V^{*}>V_{0}$. It follows that there exists a subsequence $t_{k}$, such that $\lim _{k \rightarrow \infty} E\left(t_{k}\right)=E^{*}, \lim _{k \rightarrow \infty} I\left(t_{k}\right)=I^{*}$, and $\lim _{k \rightarrow \infty} V\left(E\left(t_{k}\right), I\left(t_{k}\right)\right)=V\left(E^{*}, I^{*}\right)=V^{*}>V_{0}$. The fact that $\Delta V\left(E^{*}, I^{*}\right)<0$ and the continuity of $\Delta V(E, I)$ imply that there exists an $\eta>0$, such that $\Delta V(E, I)<\Delta V\left(E^{*}, I^{*}\right) / 2<0$ for any $(E, I)$ satisfying $\left\|(E, I)-\left(E^{*}, I^{*}\right)\right\|<\eta$. Consequently, there exists a large integer $K$ such that $\left\|\left(E\left(t_{k}\right), I\left(t_{k}\right)\right)-\left(E^{*}, I^{*}\right)\right\|<\eta$ for $k>K,\left|V\left(E\left(t_{k}\right), I\left(t_{k}\right)\right)-V\left(E^{*}, I^{*}\right)\right|<\left|\Delta V\left(E^{*}, I^{*}\right)\right| / 3$, and $\Delta V\left(E\left(t_{k}\right), I\left(t_{k}\right)\right)<\Delta V\left(E^{*}, I^{*}\right) / 2$. Then we have

$$
\begin{aligned}
V\left(E\left(t_{k+1}\right), I\left(t_{k+1}\right)\right) & =V\left(E\left(t_{k}\right), I\left(t_{k}\right)\right)+\sum_{j=t_{k}}^{t_{k+1}-1} \Delta V(E(j), I(j)) \\
& <V\left(E\left(t_{k}\right), I\left(t_{k}\right)\right)+\Delta V\left(E\left(t_{k}\right), I\left(t_{k}\right)\right) \\
& \leq V^{*}+\frac{\left|\Delta V\left(E^{*}, I^{*}\right)\right|}{3}+\frac{\Delta V\left(E^{*}, I^{*}\right)}{2} . \\
& =V^{*}+\frac{\Delta V\left(E^{*}, I^{*}\right)}{6} .
\end{aligned}
$$


Inequality $V\left(E\left(t_{k+1}\right), I\left(t_{k+1}\right)\right) \leq V^{*}+\Delta V\left(E^{*}, I^{*}\right) / 6$ implies that $\lim _{k \rightarrow \infty} V\left(E\left(t_{k}\right), I\left(t_{k}\right)\right) \leq V^{*}+$ $\Delta V\left(E^{*}, I^{*}\right) / 6$, which is a contradiction. Therefore, $\lim _{k \rightarrow \infty} V\left(E\left(t_{k}\right), I\left(t_{k}\right)\right) \leq V_{0}$, which in turn leads to

$$
\limsup _{t \rightarrow \infty} V(E(t), I(t)) \leq\left(1-p+p \alpha k_{2}+p m k_{3}\right) N^{*}\left(1-\frac{1}{R_{0}}\right)
$$

by the definition of $V_{0}$. The proof of the theorem is completed.

The requirement for $\beta_{1}$ in Theorem 4.3 is technically strict. The interval for $\beta_{1}$ should be much longer than the unit interval given in Theorem 4.3. Theorem 4.3 holds as long as $1-\beta_{1} I(t) / N^{*} \geq 0$; that is, the range of $\beta_{1}$ must guarantee that the solutions of model (4.1) are all positive.

If $0<\beta_{1} \leq 1$ and $k_{1}=0, \lim _{k \rightarrow \infty} N(t)=N^{*}$ and Theorem 4.3 implies that the solutions of model (3.1) are ultimately boundaries as $1<R_{0}<1+(1 /(1-p(1-\gamma)))$.

Theorem 4.3 provides an estimation on the ultimate boundary based on the combination of the latent and the infectious when $R_{0}>1$. We notice that most epidemic researches have focused on the persistence of the diseases by studying the existence and stability of the endemic equilibrium when $R_{0}>1$. Although we cannot obtain the exact quantity for the latent and the infectious, the ultimate boundary in Theorem 4.3 provides a novel estimation.

Figure 1(b) demonstrates the role of Theorem 4.3. The parameter values are the same as the ones we used for Figure 1(a) but with $\beta_{1}=0.3$. For that set of parameter values, $R_{0}=1.35704>1$, the endemic equilibrium $P_{1}^{*}\left(S_{1}^{*}, E_{1}^{*}, I_{1}^{*}\right)=(1228.16000,357.57414,80.93252)$, and $N^{*}=1666.66667$. The ultimate boundary domain in EI-plane is given in Figure 1(b). The largest triangle in Figure 1(b) is the feasible domain $E+I \leq N^{*}=1666.66667$. The domain under the line $V(E, I)=p \alpha k_{2} E+\left(1-p+p \alpha k_{2}+p m k_{3}\right) I=V_{0}$ is the domain of the ultimate boundary. Three solutions of model (3.1) with initial values: $E(0)=35, I(0)=200$, $E(0)=200, I(0)=1000$, and $E(0)=700, I(0)=50$, are also displayed in Figure $1(\mathrm{~b})$. Two solutions under the ultimate boundary $V(E, I)=V_{0}$ are kept below the boundary and approach the endemic equilibrium. One solution with the initial value starting outside the ultimate boundary enters the ultimate boundary domain eventually.

\subsection{With Exogenous Reinfections and Linear Treatment Rate}

\subsubsection{Backward Bifurcation}

When $k_{1}>0$, the last equation of (3.9) leads to $E_{2}^{*}=(1-p(1-\gamma)) I_{2}^{*} /\left(\left(p k_{1} \beta_{2} I_{2}^{*} / N^{*}\right)+p k_{2} \alpha\right)$. Substituting the expression $E_{2}^{*}$ into the first equation of (3.9) yields (use $I$ instead of $I_{2}^{*}$ for convenience)

$$
a_{2} I^{2}+a_{1} I+a_{0}=0
$$

where

$$
\begin{aligned}
a_{2} & =\frac{p^{2} \beta_{1} \beta_{2} k_{1}}{\left(N^{*}\right)^{2}}>0, \\
a_{1} & =\frac{p^{2} \beta_{1} k_{2} \alpha}{N^{*}}+\frac{p\left(\beta_{1}+\beta_{2} k_{1}\right)(1-p(1-\gamma))}{N^{*}}-\frac{p^{2} \beta_{1} \beta_{2} k_{1}}{N^{*}}, \\
& \triangleq-\left(R_{1}-1\right)-\left(1-R_{0}\right)\left(1+\frac{1-p(1-\gamma)-p k_{1} \beta_{2}}{p \alpha k_{2}}\right), \\
a_{0} & =(1-p(1-\gamma))\left(1-p+p k_{2} \alpha+p k_{3} m\right)-p^{2} \beta_{1} k_{2} \alpha \triangleq-c_{0}\left(R_{0}-1\right),
\end{aligned}
$$




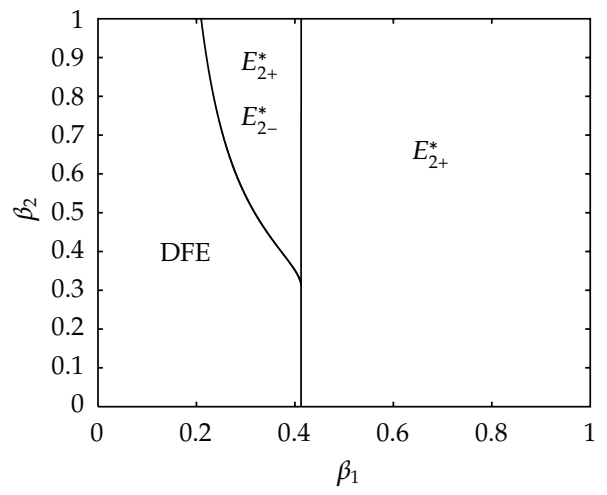

(a)

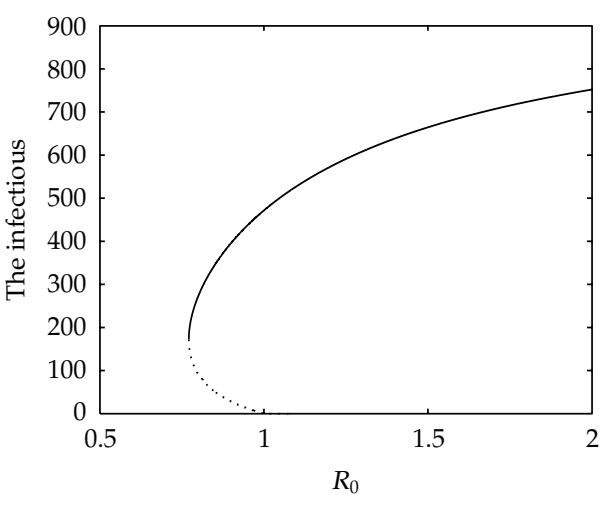

(b)

Figure 2: The backward bifurcation of model (3.9): (a) The domain with different number of endemic equilibria in $\beta_{1} \beta_{2}$-plane, (b) The bifurcation curve of model (3.9).

where

$$
\begin{array}{r}
R_{1}=\frac{p k_{1} \beta_{2}}{p \alpha k_{2}+1-p(1-\gamma)} \times \frac{1-p+p m k_{3}}{1-p+\alpha k_{2}+p m k_{3}}, \\
c_{0}=(1-p(1-\gamma))\left(1-p+p k_{2} \alpha+p k_{3} m\right)>0 .
\end{array}
$$

The type of bifurcation at $R_{0}=1$ is totally determined by $R_{1}$. Near $R_{0}=1, a_{1}<0$ holds if and only if $R_{1}>1$. Hence, at $R_{0}=1$, if $R_{1}>1$, bifurcation is forward, while if $R_{1}<1$, bifurcation is backward. $R_{1}$ gives a measure for exogenous reinfections. The first term in $R_{1}$, $1 /\left(p \alpha k_{2}+1-p(1-\gamma)\right)$, is the average re-infection period, which is a little bit shorter than the regular average infection period $1 /(1-p(1-\gamma))$. The second term in $R_{1}$ gives the proportion of the latent population. These analysis result in the following theorem that characterizes the feature of bifurcation at $R_{0}=1$.

Theorem 4.4. Consider model (3.9). If $R_{1}<1$, then the bifurcation at $R_{0}=1$ is of forward type. If $R_{1}>1$, the bifurcation at $R_{0}=1$ is of backward type.

Looking at the expression of $R_{1}$, one can see that $R_{1}>1$ necessarily requires $k_{1} \beta_{2} \neq 0$. Therefore, the exogenous reinfections are the driver behind the occurrence of the backward bifurcation. This is consistent with the continuous epidemic models for tuberculosis [30,31].

The backward bifurcation of model (3.9) is shown in Figure 2 with following parameter values $p=0.994, \Lambda=10, \alpha=0.03, \gamma=0.1, k_{1}=0.5, k_{2}=0.3, k_{3}=0.2$, and $m=0.1$. Figure $2(\mathrm{a})$ gives three domains in $\beta_{1} \beta_{2}$-plane with the unique disease-free equilibrium (DFE), two positive endemicequilibria $\left(E_{2 \pm}^{*}, I_{2 \pm}^{*}\right)$, and one endemic equilibrium $\left(E_{2+}^{*}, I_{2+}^{*}\right)$. Taking the above parameter values and fixing $\beta_{2}=0.5$, we get the backward bifurcation curve of model (3.9) (see Figure 2(b)).

We use the numerical simulation to discuss the stability of the endemic equilibria of model (3.9). The linearized matrix of (3.9) at the positive equilibrium points $P_{2 \pm}^{*}\left(E_{2 \pm}^{*} I_{2 \pm}^{*}\right)$ is

$$
J_{2 \pm}=\left(\begin{array}{cc}
p-p k_{2} \alpha-p k_{3} m-\frac{p\left(\beta_{1}+\beta_{2} k_{1}\right) I_{2 \pm}^{*}}{N^{*}} & p \beta_{1}-\frac{p\left(\beta_{1}+\beta_{2} k_{1}\right) E_{2 \pm}^{*}}{N^{*}}-\frac{2 p \beta_{1} I_{2 \pm}^{*}}{N^{*}} \\
\frac{p \beta_{2} k_{1} I_{2 \pm}^{*}}{N^{*}}+p \alpha k_{2} & \frac{p \beta_{2} k_{1} E_{2 \pm}^{*}}{N^{*}}+p(1-\gamma)
\end{array}\right) .
$$




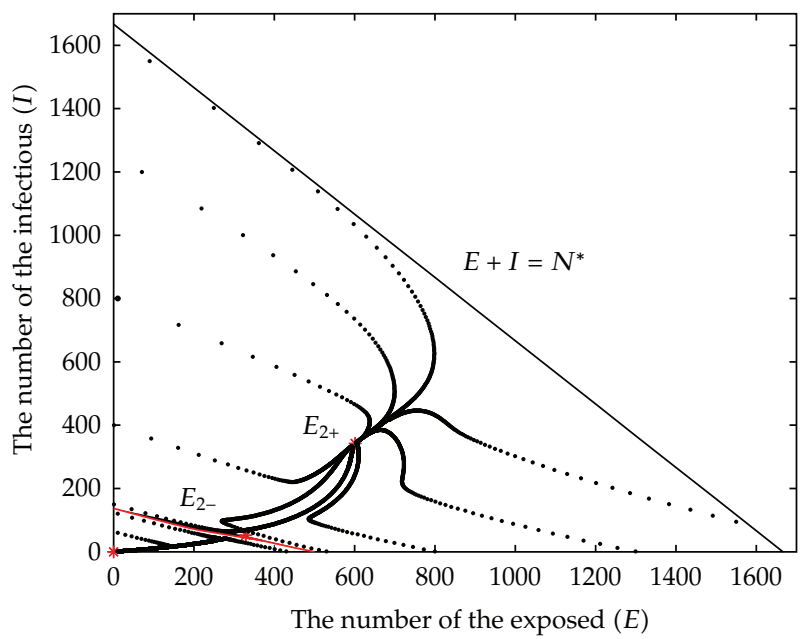

Figure 3: The attraction separatrix for those two equilibria of model (3.1).

We fix parameter values $p=0.994, \Lambda=10, \alpha=0.03, \gamma=0.1, k_{1}=0.5, k_{2}=0.3, k_{3}=0.2$, $m=0.1$, and $\beta_{2}=0.5$ and change the value of $\beta_{1}$. When $\beta_{1} \in[0.31831317735,0.41278978)$, we have $R_{0} \in[0.7711266,1)$, and there are two positive equilibrium points, which are $\left(E_{2+}^{*} I_{2+}^{*}\right)$ and $\left(E_{2-}^{*}, I_{2-}^{*}\right)$, respectively. Fixing $\beta_{1}=0.35$, we have $\left(E_{2+}^{*}, I_{2+}^{*}\right)=(601.51345,342.43485)$ and $\left(E_{2-}^{*}, I_{2-}^{*}\right)=(329.51923,52.38933)$. Accordingly, we have $\rho\left(J_{2+}\right)=0.979777<1$ and $\rho\left(J_{2-}\right)=$ $1.00415>1$. Therefore, $\left(E_{2+}^{*} I_{2+}^{*}\right)$ is local stable as $R_{0}<1$, while $\left(E_{2-}^{*} I_{2-}^{*}\right)$ is unstable as $R_{0}<1$. When $\beta_{1}>0.41278978$, we have $R_{0}>1$, and there is only one positive equilibrium point $\left(E_{2+}^{*}, I_{2+}^{*}\right)$. Fixing $\beta_{1}=1$, we have $\left(E_{2+}^{*}, I_{2+}^{*}\right)=(657.40092,796.73372)$, and $\rho\left(J_{2+}\right)=0.88069<1$. $\rho\left(J_{2+}\right)$ will change when $\beta_{1}$ becomes bigger. The numerical simulation shows that $\rho\left(J_{2+}\right)=1$ and $R_{0}=9.91134$ as $\beta_{1}=4.0913$. Therefore, we obtain that $\left(E_{2+}^{*} I_{2+}^{*}\right)$ is locally stable as $1<$ $R_{0}<9.91134$, and, when $R_{0} \geq 9.91134,\left(E_{2+}^{*}, I_{2+}^{*}\right)$ is unstable.

After spelling out the stability of the endemic equilibria, we use the numerical method to investigate the attraction domain of the positive equilibrium points in EI-plane when $R_{0}<$ 1. Here, we use the same parameter values as for the analysis of the endemic equilibrium bifurcation when $R_{0}<1$. There are three equilibrium points, the disease-free equilibrium $(0,0)$, the endemic equilibria $\left(E_{2+}^{*}, I_{2+}^{*}\right)$, and $\left(E_{2-}^{*}, I_{2-}^{*}\right)$, respectively. In Figure 3 , the solid red line is the separatrix, which divides the whole domain into two parts, the attraction domain of the larger endemic equilibrium $\left(E_{2+}^{*}, I_{2+}^{*}\right)$, and the attraction domain of the disease-free equilibrium $(0,0)$. Figure 3 shows that $\left(E_{2-}^{*} I_{2-}^{*}\right)$ is saddle, and the ultimate limit of solutions of model (3.1) depend on the initial values. If the initial values below the separatrix, the solutions will tend to the disease-free equilibrium $(0,0)$, while the solutions with initial values above the separatrix will ultimately tend to $\left(E_{2+}^{*}, I_{2+}^{*}\right)$. It appears that the attraction domain of $\left(E_{2+}^{*}, I_{2+}^{*}\right)$ is much bigger than that of $(0,0)$. This fact implies that we should be careful in assessing a disease control program, since the attraction domain of $(0,0)$ is small, the disease may not be eliminated even though $R_{0}<1$.

\subsubsection{Model Behavior for Large $R_{0}$}

This subsection is mainly concerned with the behavior of the model when $R_{0}$ is very large. Numerical approach is used to further explore the model. We fix parameter values $p=0.994$, 


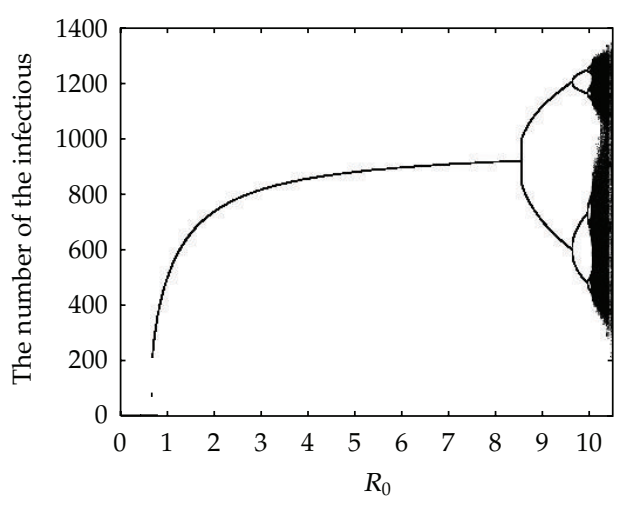

(a)

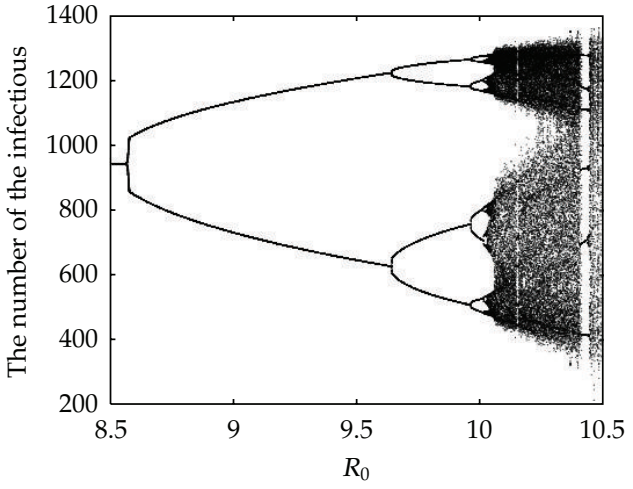

(b)

Figure 4: Period doubling bifurcation of model (3.9). Parameter values are $p=0.994, \Lambda=10, \alpha=0.03$, $r=0.1, k_{1}=0.5, k_{2}=0.3, k_{3}=0.2, m=0.1$, and $\beta_{2}=0.5$.

$\Lambda=10, \alpha=0.03, \gamma=0.1, k_{1}=0.5, k_{2}=0.3, k_{3}=0.2, m=0.1, \beta_{2}=0.5$, and let $\beta_{1}$ change. Figure 4(a) shows the existence of the stable endemic equilibrium of when $R_{0}<1$. In fact, model (3.9) has two endemic equilibrium points when $0.77113<R_{0}<1$, the small endemic equilibrium is unstable, and the large one is locally stable. If $1<R_{0}<8.6485$, model (3.9) has only one endemic equilibrium, which is locally stable. When $8.6485 \leq R_{0}<9.6417$ the endemic equilibrium of model (3.9) is unstable, and there exists a stable periodic solution of model (3.9) with period 2. Furthermore, the periodic solution with period 2 become unstable when $9.6417 \leq R_{0}<9.9566$, and a stable periodic solution with period 4 appears. When $R_{0}$ becomes larger, the period- 4 solution loses its stability and a stable period-8 solution appears (Figure $4(\mathrm{~b})$ ). The period-doubling may undergo to chaos as $R_{0}$ increases.

Figure 4 only displays the stable endemic equilibrium or stable periodic solution of model (3.9). The values of the equilibrium or the periodic solution of the infectious individuals are positive for all $R_{0} \in[0,10]$. However, the number of the latent class may be negative when $R_{0} \geq 8.6485$. $R_{0}=8.6485$ corresponds to the critical value at which the endemic equilibrium loses its stability, and periodic solution with period 2 appears. A reason for model (3.9) undergoing period-doubling solutions may be the appearance of the negative latent solutions when $R_{0}$ becomes larger. The numerical simulation seems to hint that the endemic equilibrium may be stable if $\beta_{1} \leq 1$ and $\beta_{2} \leq 1$, which gives the positive solution if the initial values are positive. Castillo-Chavez and Yakubu discussed similar problem for a discrete SIS model [19].

\subsubsection{Ultimate Boundary}

The following theorem gives an ultimate boundary to the model (3.9).

Theorem 4.5. If $1<R_{0} \leq 1+\left(\left(p \beta_{1} \alpha k_{2}-(1-p(1-\gamma))\left(1-p+p m k_{3}\right) k_{1} \beta_{2}\right) /(1-p(1-\gamma))\left(p \beta_{1} \alpha k_{2}+\right.\right.$ $\left.\left.\left(1-p+p m k_{3}\right) k_{1} \beta_{2}\right)\right), p \beta_{1} \alpha k_{2}>(1-p(1-\gamma))\left(1-p+p m k_{3}\right) k_{1} \beta_{2}$, and $0<\beta_{1}, \beta_{2} \leq 1$ hold, then the solutions of model (3.9) are ultimately bounded by

$$
\begin{aligned}
& \limsup _{t \rightarrow \infty} V(E(t), I(t))=\underset{t \rightarrow \infty}{\limsup p \alpha k_{2} E(t)+\left(1-p+p \alpha k_{2}+p m k_{3}\right) I(t) \leq V_{0}} \\
& \text { where } V^{0} \triangleq\left(1-p+p \alpha k_{2}+p m k_{3}\right) N^{*}\left(1+\frac{\left(1-p+p m k_{3}\right) k_{1} \beta_{2}}{p \beta_{1} \alpha k_{2}}-\frac{1}{R_{0}}\right) .
\end{aligned}
$$


The inequality $\lim \sup _{t \rightarrow \infty} V(E(t), I(t)) \leq V_{0}$ implies that

$$
\begin{gathered}
\limsup _{t \rightarrow \infty} E(t) \leq\left(1+\frac{1-p+p m k_{3}}{p \alpha k_{2}}\right) N^{*}\left(1+\frac{\left(1-p+p m k_{3}\right) k_{1} \beta_{2}}{p \beta_{1} \alpha k_{2}}-\frac{1}{R_{0}}\right), \\
\limsup _{t \rightarrow \infty} I(t) \leq N^{*}\left(1+\frac{\left(1-p+p m k_{3}\right) k_{1} \beta_{2}}{p \beta_{1} \alpha k_{2}}-\frac{1}{R_{0}}\right) .
\end{gathered}
$$

Introducing $V(E(t), I(t))=p \alpha k_{2} E(t)+\left(1-p+p \alpha k_{2}+p m k_{3}\right) I(t)$ and then examining the difference of $V$ yields that

$$
\begin{aligned}
\Delta V= & V(E(t+1), I(t+1))-V(E(t), I(t)) \\
= & \left(1-p(1-\gamma)\left(1-p+p \alpha k_{2}+p m k_{3}\right)\right) \\
& \times\left(R_{0}-1-\frac{R_{0}}{N^{*}} \frac{1-p+p \alpha k_{2}+p m k_{3}}{1-p+p \alpha k_{2}+p m k_{3}}(E(t)+I(t))+\frac{\left(1-p+p m k_{3}\right) k_{1} \beta_{2} R_{0} E(t)}{p \beta_{1} \alpha k_{2} N^{*}}\right) I(t) \\
\leq & \left(1-p(1-\gamma)\left(1-p+p \alpha k_{2}+p m k_{3}\right)\right) \\
& \times\left(R_{0}-1-\frac{R_{0}}{N^{*}} \frac{V(E(t), I(t))}{1-p+p \alpha k_{2}+p m k_{3}}+\frac{\left(1-p+p m k_{3}\right) k_{1} \beta_{2} R_{0}}{p \beta_{1} \alpha k_{2}}\right) I(t), \\
= & \frac{(1-p(1-\gamma)) R_{0}}{N^{*}}\left(V^{0}-V(E(t), I(t))\right) I(t) .
\end{aligned}
$$

The rest of the proof is similar to that of Theorem 4.4.

\subsection{With Exogenous Reinfections and Saturated Treatment}

In this subsection, we analyze model (3.10), that is, the SEIS model with a saturated treatment rate.

\subsubsection{Endemic Equilibria}

Let $P_{3}^{*}\left(E_{3}^{*}, I_{3}^{*}\right)$ be the positive equilibrium of model (3.10). Then the second equation of model (3.10) leads to $I_{3}^{*}=p \alpha k_{2} E_{3}^{*} /\left(1-p(1-\gamma)-\left(p \beta_{2} k_{1} E_{3}^{*} / N^{*}\right)\right)$, where $E_{3}^{*}<N^{*}(1-p(1-r)) /$ $p \beta_{2} k_{1}$.

We already have a clear picture about the equilibrium in the region $E(t) \leq E^{0}$. Now we only seek for equilibria in the region $E(t)>E^{0}$ with $T(E(t))=p\left(k_{3} E(t)-m_{0}\right)$. Similarly, $E_{3}^{*}$ satisfies

$$
\sigma_{3} E^{3}+\sigma_{2} E^{2}+\sigma_{1} E+\sigma_{0}=0
$$




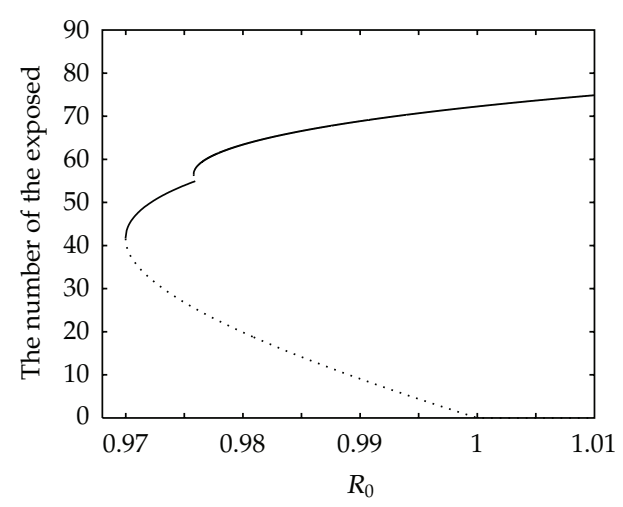

(a) Multiply Backward Bifurcation

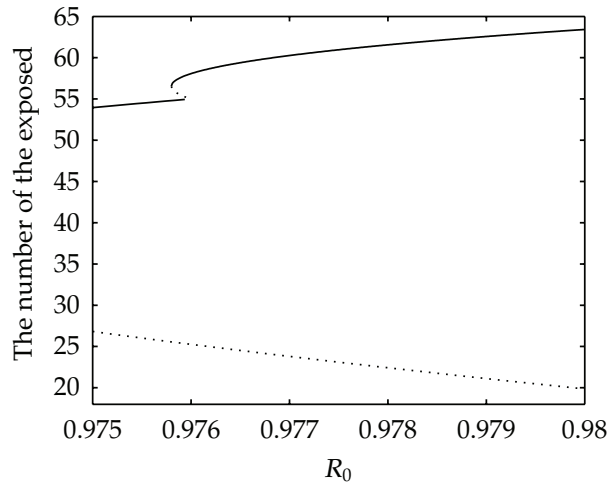

(b) A part of (a)

Figure 5: Double backward bifurcations of model (3.10). Dotted lines represent unstable equilibria, and solid lines represent stable ones.

where

$$
\begin{aligned}
\sigma_{3}= & \frac{p^{2} \beta_{2} k_{1}\left(p \beta_{1} \alpha k_{2}-\beta_{2} k_{1}(1-p)\right)}{\left(N^{*}\right)^{2}}, \\
\sigma_{2}= & \frac{2 p \beta_{2} k_{1}\left(1-p+p \alpha k_{2}\right)}{N^{*}}(1-p(1-\gamma))+\frac{p^{2} \alpha k_{2}\left(\beta_{2} k_{1}+\beta_{1}\right)}{N^{*}}(1-p(1-\gamma)) \\
& -\frac{p^{3} \beta_{1} \alpha k_{2}\left(\beta_{2} k_{1}+\alpha k_{2}\right)}{N^{*}}-\frac{p^{3} m_{0} \beta_{2}^{2} k_{1}^{2}}{\left(N^{*}\right)^{2}}, \\
\sigma_{1}= & (1-p(1-\gamma))\left(\frac{2 p^{2} m_{0} \beta_{2} k_{1}}{N^{*}}+p^{2} \beta_{1} \alpha k_{2}-(1-p(1-\gamma))\left(1-p+p \alpha k_{2}\right)\right), \\
\sigma_{0}= & -p m_{0}(1-p(1-\gamma))^{2} .
\end{aligned}
$$

From the continuity argument, we can impose $E^{0}$ to be a root to (4.27). Obviously, $R_{0}$ and $R_{1}$ are not enough to determine all possible configurations of the roots of (4.27). We can directly use these sigmas that have less epidemiological meaning. Notice that $\sigma_{0}<0$ relieves our work a little bit. If $\sigma_{3}<0$, then (4.27) has exact two positive roots. If $\sigma_{3}>0$ and $\sigma_{1}<0$, then (4.27) has exact one positive root. If $\sigma_{3}>0$ and $\sigma_{1}>0$, then (4.27) has exact one or three positive roots. For all situations, $E^{0}$ is always the smallest positive root.

Next we will explore the dynamics of model (3.10) numerically by carefully selecting parameter values.

\subsubsection{Double Backward Bifurcations}

A double backward bifurcation is observed for parameter values $p=0.994, \Lambda=10, k_{1}=0.2$, $k_{2}=0.4, k_{3}=0.4, \alpha=0.03, \gamma=0.0001, m=0.005$, and $\beta_{2}=0.375$.

As can be seen in Figure 5, a backward bifurcation occurs at $R_{0}=1$ because $R_{1}=$ $1.6527>1$. This backward bifurcation is bifurcated from the disease-free equilibrium. 


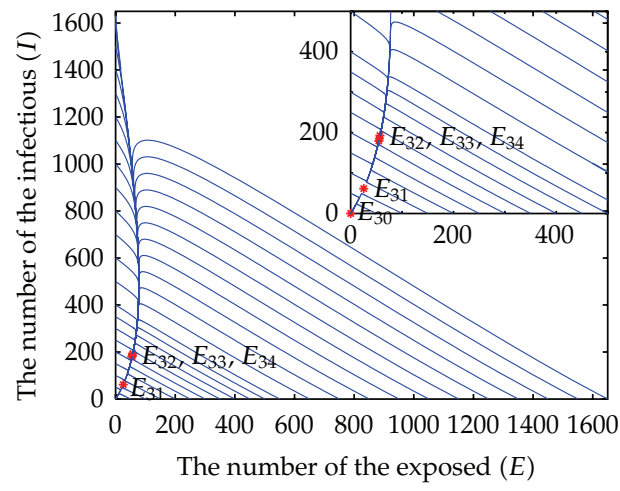

(a)

Figure 6: Phase portrait of model (3.10): a case of multiple equilibria.

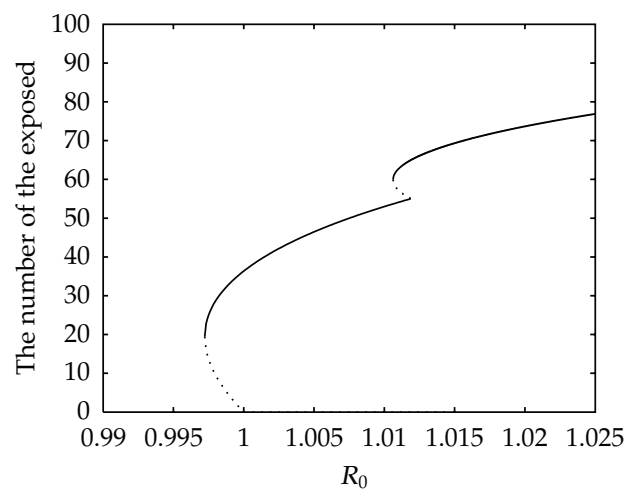

(a) Multiply backward bifurcation

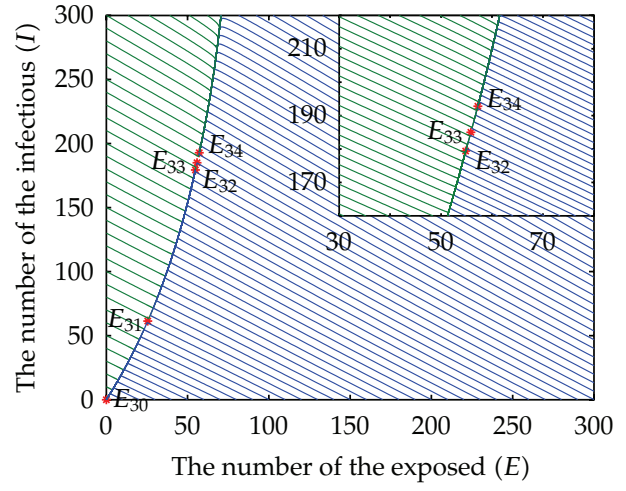

(b)

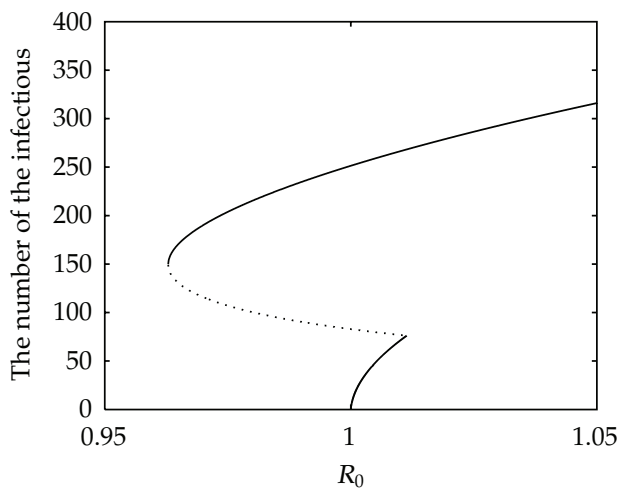

(b) Forward-backward bifurcation

Figure 7: (a) Double backward bifurcations for model (3.10). The upper one occurs when $R_{0}>1$. (b) Forward-backward bifurcation for model (3.10). The upper one occurs when $R_{0}<1$. Dotted lines represent unstable equilibria, and solid lines represent stable ones.

Meanwhile, at an endemic equilibrium, the second backward bifurcation happens, so that we can see that there is a window of five equilibria. Three of them are stable, and two of them are unstable. Indeed, we have found a tristability when $R_{0}<1$ and when saturated treatment rate is used. Figure 6 shows the tristable situation on the phase plane.

If we choose $\beta_{2}=0.26$, then $R_{1}=1.1458>1$. The bifurcation at $R_{0}=1$ remains backward. However, the second backward bifurcation shifts to the right, happening at a place of $R_{0}>1$. One can observe bistability not only for $R_{0}<1$ but also for $R_{0}>1$. Figure 7(a) illustrates this bistable dynamics. Further decreasing of $\beta_{2}$ can produce a new bifurcation, which will be studied in the next subsection.

\subsubsection{Forward-Backward Bifurcation}

If we set $p=0.994, \Lambda=8, k_{1}=0.3, k_{2}=0.3, k_{3}=0.4, \alpha=0.03, \gamma=0.001, m=0.01$, $m_{0}=0.2$, and $\beta_{2}=0.1$, then the bifurcation at $R_{0}=1$ is forward because $R_{1}=0.9835<1$, as we can see from Figure $7(b)$ that a backward bifurcation occurs on the top. The backward 


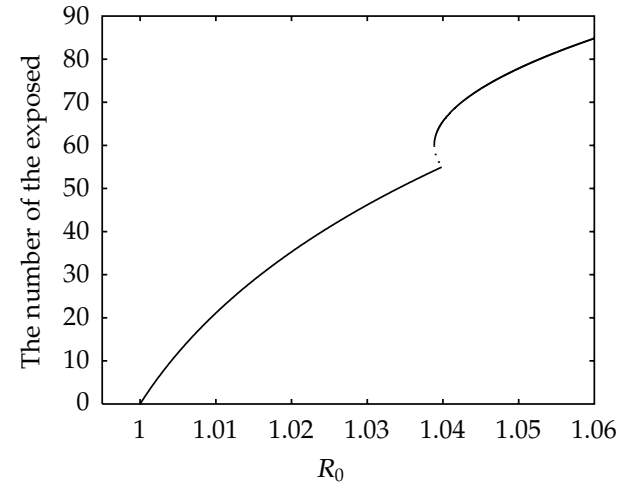

(a) Forward-backward bifurcation

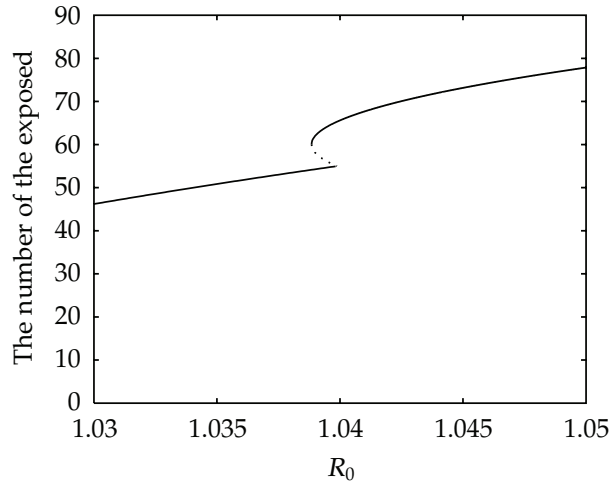

(b) A part of (a)

Figure 8: Forward-backward bifurcation for model (3.10). The upper one occurs when $R_{0}>1$. Dotted lines represent unstable equilibria, and solid lines represent stable ones.

bifurcation curve returns all the way back to the region of $R_{0}<1$, generating an endemic in the region. This would be the worst consequence of the saturated treatment strategy because it help to establish the disease when $R_{0}<1$. If a fixed proportion of the latent individuals are treated (linear treatment rate is used), then the backward bifurcation on the top cannot happen, consequently the disease dies out when $R_{0}<1$.

A less important forward-backward bifurcation is the case where the whole backward bifurcation curve exists only in the region of $R_{0}>1$, thus only making endemic equilibrium bigger. Figure 8 shows this kind of bifurcation, where $\beta_{2}=0.18$ in order to make $R_{1}=0.7933<$ 1 and the rest of parameters are the same as in the previous subsection.

\section{Conclusion and Discussion}

In this paper, we analyzed a class of discrete SEIS models and their epidemiological implications. First, the models without exogenous reinfections were considered. We obtained the global stability of the disease-free equilibrium, existence and uniqueness of endemic equilibrium and its stability. We also found the sufficient conditions for the ultimate boundary of the solutions. Then we considered the models with exogenous reinfections. These models undergo a backward bifurcation. Furthermore, we showed that the models with exogenous re-infection and treatment exhibit period-double phenomenon when $R_{0}$ grows larger. The period-double bifurcation was obtained for the reduced model (3.9). One component in the full model may become negative when the reduced model has periodic orbits. Finally we considered the models with exogenous re-infection and saturated treatment. No matter whether there is the exogenous re-infection, the saturated treatment can lead to a backward bifurcation at an endemic, but exogenous re-infection determines the backward bifurcation at the disease-free equilibrium.

From our qualitative and quantitative analysis, we had already found that a combination of exogenous reinfections and treatment regimens is capable of generating complex dynamics. These include tristability (disease-free equilibrium and two endemic equilibria) when $R_{0}<1$, bistability for both $R_{0}<1$ and $R_{0}>1$, and period doubling for a reduced model.

We now know that $k_{1} \beta_{2}$ controls the appearance of the backward bifurcation at $R_{0}=1$, creating an endemic equilibrium when $R_{0}<1$, an example of bistable scenario. The treatment 
regime does not participate in the backward bifurcation $R_{0}=1$. However, the treatment regime is able to create equilibria at a higher level, far away from the disease-free equilibrium, regardless of the values of $R_{0}$. The treatment strategy change actually means stopping the trend of treatment effort and treating fewer individuals. This reduction in treatment efforts leads to the emerging of the larger endemic equilibrium. The worst scenario is that if the bifurcation at $R_{0}=1$ is forward, meaning that $R_{0}<1$ makes the disease die out, and if the treatment practice is changed through reducing the treatment effort, then an endemic is born when $R_{0}<1$. This reduction of treatment effort helps to reestablish the disease at local level, making it impossible to eliminate the disease. A plausible conclusion is the strategy should be to treat as many latent individuals as possible. Our model supports that the strategy that a fixed percentage (proportion) of the latent individuals should be treated consistently to eliminate the disease. For diseases like tuberculosis, failure to take care of the existence of long-lived latent individuals may be a critical reason to the longevity of the disease. Exogenous reinfections intrinsically result in bistability through the occurrence of a backward bifurcation, which is out of our control. However, persistence of the disease resulting from a change of policy should and can be avoided by all means. Obviously, it would be a sad story if reduction of treatment is due to an insufficient health infrastructure or budget.

\section{Acknowledgment}

This study was supported by NSFC (10971163) and by IDRC of Canada (104519-010).

\section{References}

[1] R. M. May, "Biological populations obeying difference equations: stable points, stable cycles, and chaos," Journal of Theoretical Biology, vol. 51, no. 2, pp. 511-524, 1975.

[2] R. M. May, “Deterministic models with chaotic dynamics," Nature, vol. 256, no. 5514, pp. 165-166, 1975.

[3] R. M. May, "Simple mathematical models with very complicated dynamics," Nature, vol. 261, no. 5560, pp. 459-467, 1976.

[4] M. P. Hassell, "Density dependence in single-species populations," Journal of Animal Ecology, vol. 44, pp. 283-289, 1975.

[5] C. Castillo-Chavez and A.-A. Yakubu, "Discrete-time SIS models with complex dynamics," Nonlinear Analysis, vol. 47, no. 7, pp. 4753-4762, 2001.

[6] C. Castillo-Chavez and A. A. Yakubu, "Dispersal, disease and life-history evolution," Mathematical Biosciences, vol. 173, no. 1, pp. 35-53, 2001.

[7] J. Dushoff, W. Huang, and C. Castillo-Chavez, "Backwards bifurcations and catastrophe in simple models of fatal diseases," Journal of Mathematical Biology, vol. 36, no. 3, pp. 227-248, 1998.

[8] H. Zhu, S. A. Campbell, and G. S. K. Wolkowicz, "Bifurcation analysis of a predator-prey system with nonmonotonic functional response," SIAM Journal on Applied Mathematics, vol. 63, no. 2, pp. 636-682, 2002.

[9] J. N. Cui, X. X. Mu, and H. Wan, "Saturation recovery leads to multiple endemic equilibria and backford bifurcation," Journal of Theoretical Biology, vol. 254, pp. 275-283, 2008.

[10] K. P. Hadeler and P. van den Driessche, "Backward bifurcation in epidemic control," Mathematical Biosciences, vol. 146, no. 1, pp. 15-35, 1997.

[11] P. van den Driessche and J. Watmough, "A simple SIS epidemic model with a backward bifurcation," Journal of Mathematical Biology, vol. 40, no. 6, pp. 525-540, 2000.

[12] S. M. Moghadas, "Analysis of an epidemic model with bistable equilibria using the Poincare index," Applied Mathematics and Computation, vol. 149, no. 3, pp. 689-702, 2004.

[13] W. Wang, "Backward bifurcation of an epidemic model with treatment," Mathematical Biosciences, vol. 201, no. 1-2, pp. 58-71, 2006.

[14] Y. Xiao and S. Tang, "Dynamics of infection with nonlinear incidence in a simple vaccination model," Nonlinear Analysis, vol. 11, no. 5, pp. 4154-4163, 2010. 
[15] X. Z. Li, W. S. Li, and M. Ghosh, "Stability and bifurcation of an SIR epidemic model with nonlinear incidence and treatment," Applied Mathematics and Computation, vol. 210, no. 1, pp. 141-150, 2009.

[16] L. J. S. Allen, "Some discrete-time SI, SIR, and SIS epidemic models," Mathematical Biosciences, vol. 124, no. 1, pp. 83-105, 1994.

[17] L. J. S. Allen and A. M. Burgin, "Comparison of deterministic and stochastic SIS and SIR models in discrete time," Mathematical Biosciences, vol. 163, no. 1, pp. 1-33, 2000.

[18] L. Allen, D. Flores, R. Ratnayake, and J. Herbold, "Discrete-time deterministic and stochastic models for the spread of rabies," Applied Mathematics and Computation, vol. 132, no. 2-3, pp. 271-292, 2002.

[19] C. Castillo-Chavez and A. A. Yakubu, "Discrete-time SIS models with simple and complex population dynamics," in Mathematical Approaches for Emerging and Reemerging Infectious Diseases: An Introduction, C. Castillo-Chavez, S. Blower, P. van den Driessche, D. Kirschner, and A. A. Yakubu, Eds., vol. 125, pp. 153-163, Springer, New York, NY, USA, 2002.

[20] R. Arreola, A. Crossa, M. C. Velasco, and A. A. Yakubu, "Discrete-time SEIS models with exogenous re-infection and dispersal between two patches," http://mtbi.asu.edu/files/0835_001.pdf .

[21] P. A. Gonzalez, R. A. Saenz, B. N. Sanchez, C. Castillo-Chavez, and A. A. Yakubu, “Dispersal between two patches in a discrete time SEIS model," MTBI Technical Report, Cornell University, 2000.

[22] J. E. Franke and A.-A. Yakubu, "Discrete-time SIS epidemic model in a seasonal environment," SIAM Journal on Applied Mathematics, vol. 66, no. 5, pp. 1563-1587, 2006.

[23] Z. Yicang and P. Fergola, "Dynamics of a discrete age-structured SIS models," Discrete and Continuous Dynamical Systems. Series B, vol. 4, no. 3, pp. 843-852, 2004.

[24] Y. C. Zhou and Z. Ma, "Global stability of a class of discrete age-structured SIS models with immigration," Mathematical Biosciences and Engineering, vol. 6, no. 2, pp. 409-425, 2009.

[25] Y. C. Zhou and H. Cao, "Discrete tuberculosis transmission models and their application," in A Survey of Mathematical Biology, S. Sivaloganathan, Ed., vol. 57 of Fields Communications Series, pp. 83-112, A co-publication of the AMS and Fields Institute, Canada, 2010.

[26] H. R. Thieme, "Uniform persistence and permanence for non-autonomous semiflows in population biology," Mathematical Biosciences, vol. 166, no. 2, pp. 173-201, 2000.

[27] H. R. Thieme, "Persistence under relaxed point-dissipativity (with application to an endemic model)," SIAM Journal on Mathematical Analysis, vol. 24, no. 2, pp. 407-435, 1993.

[28] G. Butler and P. Waltman, "Persistence in dynamical systems," Journal of Differential Equations, vol. 63, no. 2, pp. 255-263, 1986.

[29] P. Waltman, "A brief survey of persistence in dynamical systems," in Delay Differential Equations and Dynamical Systems, S. Busenberg and M. Martelli, Eds., vol. 1475 of Lecture Notes in Mathematics, pp. 31-40, Springer, Berlin, Germany, 1991.

[30] Z. Feng, C. Castillo-Chavez, and A. F. Capurro, "A model for tuberculosis with exogenous reinfection," Theoretical Population Biology, vol. 57, no. 3, pp. 235-247, 2000.

[31] C. Castillo-Chavez and B. Song, "Dynamical models of tuberculosis and their applications," Mathematical Biosciences and Engineering, vol. 1, no. 2, pp. 361-404, 2004.

[32] B. Song and C. Castillo-Chavez, "Tuberculosis control in the U.S.A.: a strategy to meet CDC's goal," Department of Biometrics Technical Report series BU-1562-M, Biometrics Department, Cornell University.

[33] E. Ziv, C. L. Daley, and S. M. Blower, "Early therapy for latent tuberculosis infection," American Journal of Epidemiology, vol. 153, no. 4, pp. 381-385, 2001.

[34] L. Allen and P. van den Driessche, "The basic reproduction number in some discrete-time epidemic models," Journal of Difference Equations and Applications, vol. 14, no. 10-11, pp. 1127-1147, 2008.

[35] X. Q. Zhao, "Asymptotic behavior for asymptotically periodic semiflows with applications," Communications on Applied Nonlinear Analysis, vol. 3, no. 4, pp. 43-66, 1996.

[36] S. N. Elaydi, An Introduction to Difference Equations, Undergraduate Texts in Mathematics, Springer, New York, NY, USA, 2nd edition, 1999. 


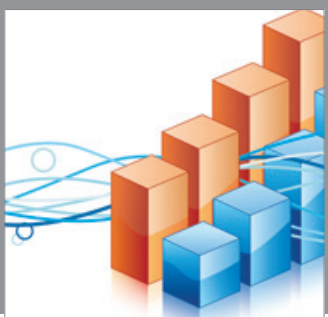

Advances in

Operations Research

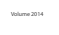

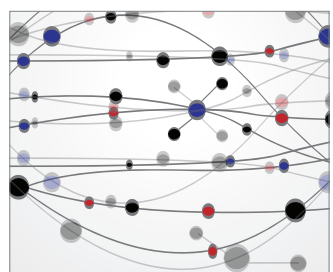

\section{The Scientific} World Journal
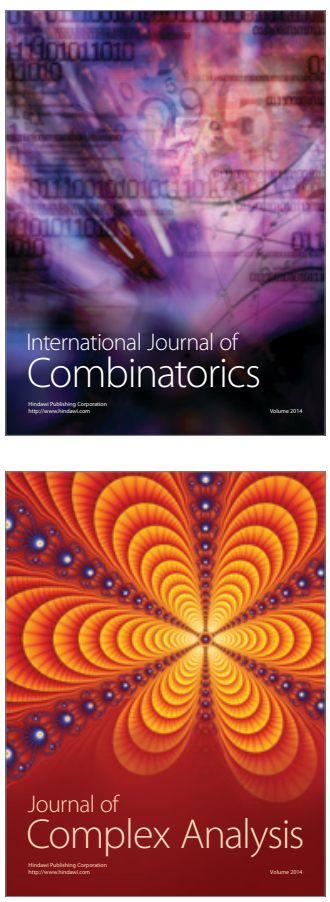

International Journal of

Mathematics and

Mathematical

Sciences
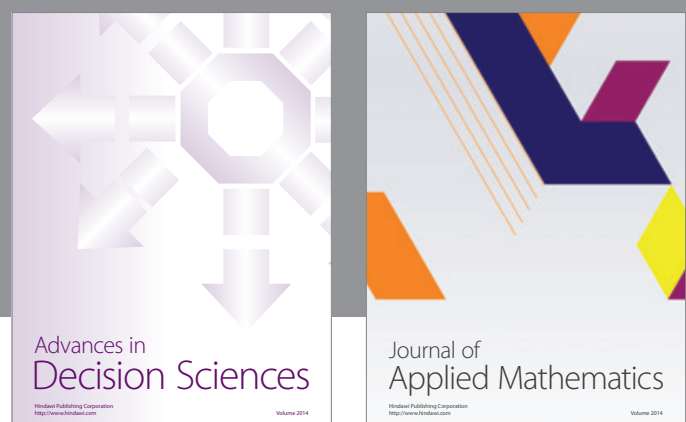

Journal of

Applied Mathematics
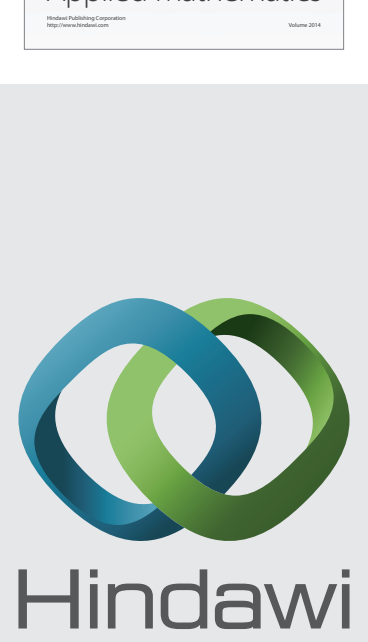

Submit your manuscripts at http://www.hindawi.com
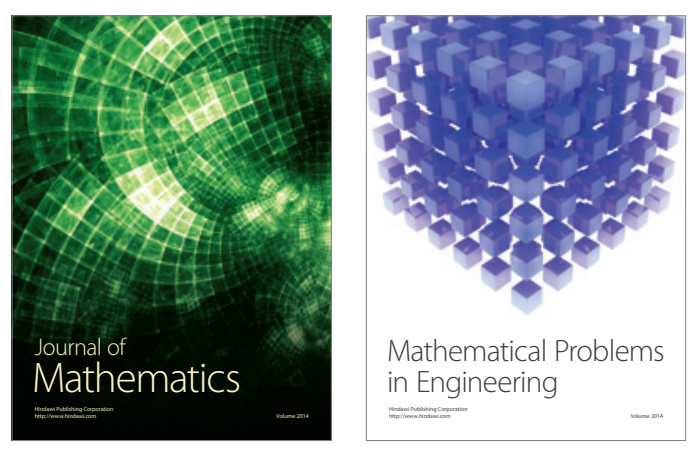

Mathematical Problems in Engineering
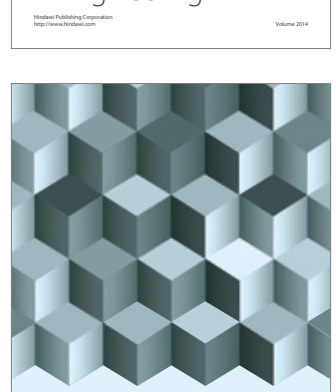

Journal of

Function Spaces
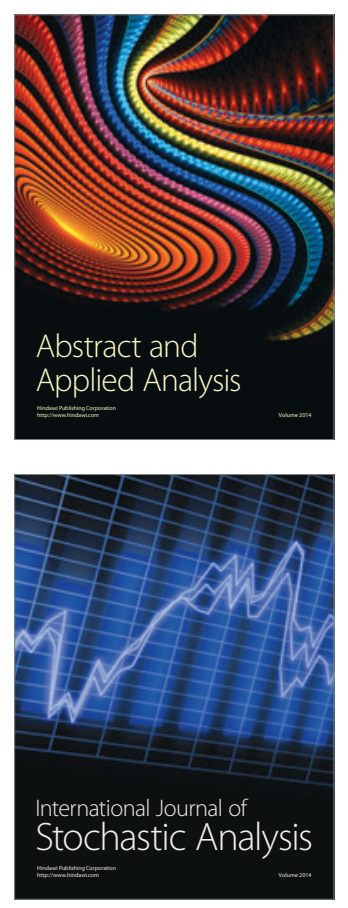

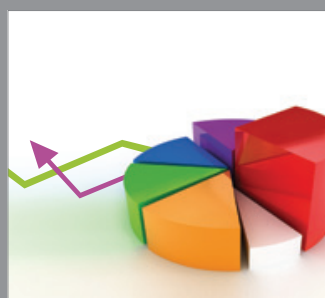

ournal of

Probability and Statistics

Promensencen
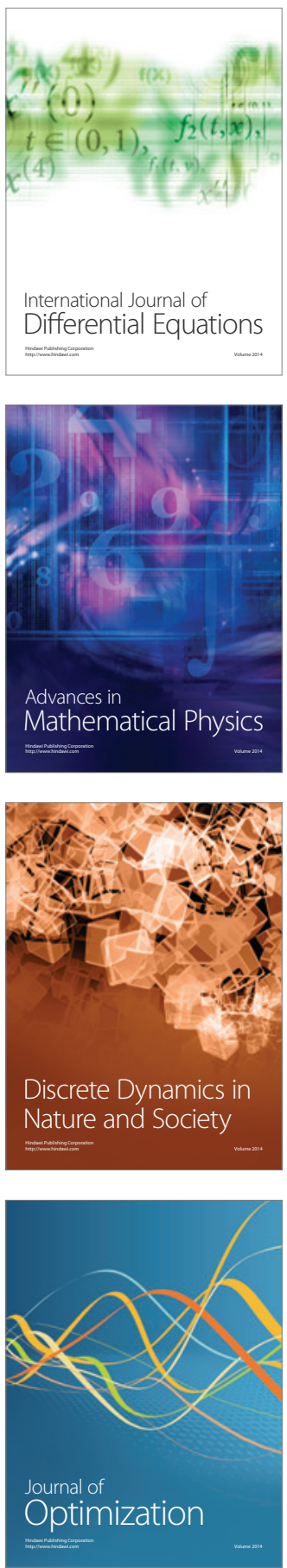